\title{
Caracterización de la actividad amilásica presente en extractos larvarios de dos polillas plagas de la papa: Tecia solanivora y Symmetrischema tangolias
}

\author{
Patricia Mora-Criollo, Andrea Rodríguez-Guerra y Carlos A. Soria \\ Laboratorio de Bioquímica, Escuela de Ciencias Biológicas, Pontificia Universidad Católica del Ecuador, \\ Quito, Ecuador \\ casoria@puce.edu.ec
}

Recibido: 11, 05, 2013; aceptado: 09, 10, 2013

RESUMEN.- Las polillas Tecia solanivora y Symmetrischema tangolias (Lepidoptera: Gelechiidae) ocasionan daños significativos a los tubérculos de Solanum tuberosum. El objetivo de este estudio fue aislar y caracterizar bioquímicamente las amilasas presentes en los diferentes estadios larvales de estas polillas. Para efectos comparativos se extrajo las proteínas solubles de estadios larvales criados en el laboratorio para determinar espectrofotométricamente diferencias en la concentración de cada extracto. Se calculó el peso promedio de cada larva: T. solanivora resultó ser más pesada y de ella se obtuvo más proteína soluble en comparación con $S$. tangolias. La actividad amilásica en los extractos proteicos fue identificada mediante degradación de almidones. Extractos de los estadios IV de ambas polillas, incubados a diferentes intervalos de tiempo, presentaron actividades amilásicas diferentes, aunque resultaron bastante similares cuando se leyeron los resultados de cada extracto tarde a las $72 \mathrm{~h}$. Mediante electroforesis de los extractos proteicos de las larvas de las dos especies, migraron alrededor de 11 bandas proteicas entre 225 y $10 \mathrm{kDa}$. Entre estas bandas, las amilasas fueron reconocidas en ambas especies y en los 4 estadios como bandas de $50 \mathrm{kDa}$. La(s) banda(s) probablemente isofórmicas de esta enzima aparecieron muy definidas en los estadios I y II, en contraste con las formas difusas encontradas en los estadios III y IV. La actividad amilásica no estuvo ligada a las concentraciones proteicas sino a las condiciones de reacción. El estudio sugiere la posibilidad de diseñar formas inéditas de biocontrol contra estas plagas.

PALABRAS CLAVES: almidón, amilasa, electroforesis, espectrofotometría, polillas

ABSTRACT.- Tecia solanivora and Symmetrischema tangolias (Lepidoptera: Gelechiidae) moths cause serious damage to Solanum tuberosum tubercles. The purpose of this study was to isolate and biochemically characterize amilases present in different larval moth stages. Soluble proteins were extracted from the larval stages raised in the laboratory. Differences in protein concentration were determined by spectrophotometry. Average weight for each larvae was calculated. T. solanivora was heavier and more soluble protein 
was extracted as compared with S. tangolias. Amilase activity of the different protein extracts was identified using starch degradation techniques. The amylasic activity of the IV stage of $T$. solanivora and $S$. tangolias incubated at different intervals of time were found to react different, however the results became similar when the activity of both extracts was scored after $72 \mathrm{~h}$ time reaction. Eleven protein bands between 225 and 10 $\mathrm{kDa}$ were identified electrophoretically. Among these bands, amylases were identified in both species in the 4 different stages in accordance to the molecular weights equivalent to $50 \mathrm{kDa}$. The protein bands of this enzyme were more defined in the I and II than in the III and IV stages, where they appeared quite diffused. Amylase activity was not linked to soluble protein concentrations; instead, it depended on reaction conditions. These results suggest the possibility of designing new forms of biocontrol against potato moths.

KEYWORDS: amylase, electrophoresis, moths, spectrophotometry, starch

\section{INTRODUCCIÓN}

Las polillas Tecia solanivora y Symmetrischema tangolias (Lepidoptera: Gelechiidae) son plagas capaces de ocasionar daños muy significativos a los tubérculos de Solanum tuberosum. Se estima que estas polillas, sin un control adecuado, pueden dañar hasta un $30 \%$ de los tubérculos del campo y hasta un $50 \%$ de los que se encuentran almacenados en bodegas (Domínguez et al., 2009).

T. solanivora, considerada la más agresiva de las dos plagas citadas, ha migrado desde América Central hasta Ecuador, e incluso ha sido reportada en las Islas Canarias en España (Trujillo et al., 2002), por lo cual es considerada una plaga de importancia económica que podría continuar ampliando su distribución (Valderrama et al., 2007). La otra especie, $S$. tangolias es una plaga andina de mayor distribución e igualmente ocasiona pérdidas económicas importantes entre los cultivadores de papa (Lagnaoui et al., 2000).

La alimentación voraz de las polillas en su estado larvario, provoca considerables daños que se observan a simple vista en el tubérculo, por lo cual asumimos que cada estadio despliega diferentes grados de actividad enzimática, acompañada de cambios proteicos estructurales y funcionales que se identifican con el modelo de infestación. Algunas clases de insectos tienen una dieta rica en almidones los cuales deben ser procesados por enzimas como las amilasas (Kotkar et al., 2012; Viktorinova et al., 2011;). La $\alpha$-amilasa (alfaamilasa 1.4-glucan-4-glucanoanhidrasa), ha sido descrita en los órdenes: Diptera, Coleoptera, Hymenoptera, Lepidoptera y Hemiptera y tiene la función de catalizar la hidrólisis de las uniones $\alpha-1.4$ glucosídicas (Darvishzadeh et al., 2012; Ngernyuang et al., 2010; Kazzazi et al., 2005; Baker, 1983). El 
estudio del funcionamiento de estas enzimas y la relación con la estructura y función proteica del insecto, permiten comprender los mecanismos de infestación empleados por estas plagas en sus diferentes estadios.

Nuestro objetivo fue aislar proteínas larvarias hidrosolubles, separarlas, determinar diferencias entre estadios y entre especies e identificar enzimas como la amilasa en cada estadio larvario mediante técnicas de espectrofotometría, geles de poliacrilamida y medios para determinar la duración de la actividad amilásica extracelular a diferentes intervalos de tiempo.

\section{MATERIALES Y MÉTODOS}

Crianza de las polillas.- T. solanivora y S. tangolias fueron criadas en el Laboratorio de Control Biomolecular de plagas de la Pontificia Universidad Católica del Ecuador. Los adultos, machos y hembras, fueron colocados en cámaras de cópula según protocolo previamente descrito por Barragán (2005) y Herrera (2010) con modificaciones importantes. Se usaron tubérculos de la variedad Cecilia, de aproximadamente $40 \mathrm{~g}$ de peso cada uno; los huevos ovipositados en pedazos de cartulina (10 aproximadamente), se colocaron sobre la superficie de cada tubérculo. La cámara experimental de infección fue incubada a la sombra a $18^{\circ}$ C, donde permaneció por varios días hasta el desarrollo del estadio de interés. Las larvas fueron mecánicamente extraídas y colectadas asépticamente en el tiempo requerido para el desarrollo de cada estadio en cada especie, a partir de la puesta del huevo, según como se indica en la Tabla 1.

Tabla 1

Períodos de desarrollo larvario a partir de la oviposición (en días, a $18^{\circ} \mathrm{C}$ )

\begin{tabular}{cccc}
\multicolumn{2}{c}{ Tecia solanivora } & \multicolumn{2}{c}{$\begin{array}{c}\text { Symmetrischema } \\
\text { tangolias }\end{array}$} \\
Estadios & Días & Estadios & Días \\
I & 20 & I & 20 \\
II & 25 & II & 28 \\
III & 33 & III & 35 \\
IV & 41 & IV & 42 \\
\hline
\end{tabular}

Peso y preparación de los extractos larvarios.- Las larvas colectadas pertenecientes a un determinado estadio y especie fueron contadas individualmente, luego pesadas en una balanza de precisión (Boeco BBI-31) hasta obtener un peso total promedio de $120 \mathrm{mg}$. Las larvas fueron maceradas y homogenizadas durante $5 \mathrm{~min}$ en $0.6 \mathrm{ml}$ (proporción 1:5 p/v) de tampón fosfato ( $\mathrm{pH}$ 7.8) mediante tubos Eppendorf de 1.5 mly pistilos estériles para cada especie y para cada estadio. Los homogenizados fueron centrifugados durante $15 \mathrm{~min}$ a 13 $000 \mathrm{rpm}$ en una centrífuga Spectrafuge 24D (Labnet). Se recuperó el total de $0.5 \mathrm{ml}$ de los sobrenadantes de cada estadio en 
cada tubo por separado. Los tubos fueron identificados como extractos de proteína soluble en buffer fosfato los mismos que fueron almacenados a $4^{\circ} \mathrm{C}$ para análisis posteriores.

Cuantificación proteica total.- Se analizó la concentración proteica soluble de cada extracto mediante el uso del kit de cuantificación QuantiPro ${ }^{\mathrm{TM}}$ BCA Sigma, con modificaciones implementadas en el laboratorio (Rodríguez-Guerra et al., 2012; Astudillo y Soria, 2011). Los reactivos se prepararon mezclando $20 \mu \mathrm{l}$ de la muestra de extracto diluido 1:100 con $20 \mu \mathrm{l}$ del reactivo de cuantificación (dilución final 1:200). Los tubos fueron incubados a $60^{\circ} \mathrm{C}$ por una hora; posteriormente se procedió a medir la absorbancia a $562 \mathrm{~nm}$ con un espectrofotómetro (Nanodrop Thermo Scientific). Las absorbancias de los extractos fueron convertidas a concentraciones proteicas en base a una curva estándar de albúmina de suero bovino (BSA) elaborada mediante concentraciones que fluctuaron entre 0 hasta $0.5 \mathrm{mg}$ de albúmina por $\mathrm{ml}$.

\section{Determinación cualitativa de} la actividad amilásica.- La prueba se realizó en medio Agar-Papa preparado con 29 g de papa de la variedad Cecilia, previamente cocida por 5 min a ebullición en $500 \mathrm{ml}$ de agua destilada más $7.5 \mathrm{~g}$ de Agar-Agar (Himedia). El medio fue autoclavado por $1 \mathrm{~h}$, luego repartido en cajas de Petri (20 ml por placa) bajo una cabina de flujo laminar (ESCO Optimair). El estudio de la actividad amilásica se llevó a cabo siguiendo la metodología de Rodríguez-Guerra et al., (2012) con algunas modificaciones. Cincuenta $\mu \mathrm{ldel}$ extracto obtenido de cada estadio fueron incorporados de forma estéril en un orificio central $(6 \mathrm{~mm}$ de diámetro por 4 $\mathrm{mm}$ de profundidad) que se creó con un sacabocados en el medio de cultivo de una caja de Petri. Los halos de actividad que se formaron a $18^{\circ} \mathrm{C}$ fueron analizados, fotografiados y cuantificados a las 8, 24, 48 y 72 h después de la inoculación y después de haber sido coloreados con una solución oxidante de lugol ( 2 g de KI, $1 \mathrm{~g}$ de I en 100 $\mathrm{ml}$ de $\mathrm{H}_{2} \mathrm{O}$ ). Cada experimento se realizó por triplicado.

Se efectuaron dos comparaciones de la actividad amilásica: la primera, entre diferentes intervalos y el estadio larvario (IV) y la segunda, una comparación entre todos los estadios larvarios y un solo intervalo de tiempo (72 h).

Separación e identificación de las proteínas solubles por electroforesis.- La caracterización proteica de los extractos se llevó a cabo en geles de poliacrilamida con SodioDodecilSulfato(SDS-PAGE), seutilizó el protocolo estándar con modificaciones de Astudillo y Soria (2011), Kazzazi (2005) y Campos (1989), para la identificación de amilasas. Los geles fueron preparados al 12 $\%$, las muestras fueron desnaturalizadas por 15 min en baño maría a ebullición. A continuación, se colocaron $15 \mu \mathrm{l}$ de estas muestras procesadas en cada pocillo con inclución de un pocillo de referencia con una escalera molecular Promega V849A (10 $\mu \mathrm{l})$, que permitió visualizar rangos de 
pesos moleculares de las bandas proteicas en kilodaltons ( $\mathrm{kDa})$. El proceso se llevó a cabo en una cámara de electroforesis Fisher Scientific FB-VE10-1, a $100 \mathrm{~V}$ durante 3 horas en tampón $1 \mathrm{X}$ de TRIS/HCL, $\mathrm{pH}$ 8.3. Las bandas se visualizaron mediante tinción conteniendo azul de Coomassie al $1 \%$, por $1 \mathrm{~min}$ a $92^{\circ} \mathrm{C}$ seguido de una agitación moderada y continua por 10 min. Se fijaron las muestras en búfer de des tinción calentado a $92^{\circ} \mathrm{C}$ por 1 min ó hasta que las bandas sean observadas claramente.

Análisis estadístico.- Se realizó un ANOVA de una vía, con diseño aleatorio para las 3 lecturas de medición de los halos de cada uno de los 4 intervalos de tiempo de los estadios larvarios y un ANOVA factorial $2 \times 4$, de las concentraciones proteicas entre las dos especies de proteína soluble total y proteína soluble por larva. Adicionalmente, se realizó un análisis de correlación de los datos de la actividad amilásica a las $72 \mathrm{~h}$ de actividad y la concentración proteica entre las dos especies.

\section{RESULTADOS}

Peso de las larvas y cuantificación proteica total.- Se contó un número determinado de larvas de cada estadio hasta alcanzar un peso estándar equivalente a $120 \mathrm{mg}$; se calculó el peso promedio en mg para las larvas de cada estadio de cada especie (Tabla 2). Las larvas de $T$. solanivora resultaron ser progresivamente más pesadas en cada nuevo estadio, se necesitaron menos larvas de $T$. solanivora comparadas con las de $S$. tangolias para llegar a los $120 \mathrm{mg}$ de peso de cada estadio para estudios proteicos comparativos.

Tabla 2

Períodos de desarrollo larvario a partir de la oviposición (en días, a $18^{\circ} \mathrm{C}$ )

Tecia solanivora

No. de larvas

(120 mg/estadio; $\left.\mathrm{s}^{*}\right)$

\begin{tabular}{cc} 
Estadio & \\
\hline I & $42 \pm 0.96$ \\
II & $28 \pm 0.58$ \\
III & $5 \pm 0.58$ \\
IV & $3 \pm 0.5$
\end{tabular}

Peso (mg)/larva; s*

$2.84 \pm 0.06$

$4.36 \pm 0.09$

$27.00 \pm 3.46$

$45.00 \pm 10$
Symmetrischema tangolias

No. De larvas

(120 mg/estadio; s*) Peso (mg)/larva; s *

$\begin{array}{ccc}65 \pm 0.50 & & 1.84 \pm 0.02 \\ 42 \pm 0.50 & & 2.85 \pm 0.03 \\ 7 \pm 0.5 & & 17.14 \pm 1.43 \\ 5 \pm 0.5 & & 24.00 \pm 3\end{array}$

$\mathbf{s}^{*}=$ desviación estándar

Se observaron diferencias en las conforme maduraban los estadios concentraciones proteicas totales de cada extracto y de cada larva, determinadas por espectrofotometría (Tabla 3 y 4). Los larvarios. La menor concentración proteica soluble se observó en el extracto diluido 1:200 (0.072 mg/ml) y en la de cada larva valores de los extractos proteicos de $T$. solanivora aumentaron progresivamente (0.17 mg/larva) del estadio I, en contraste con las concentraciones significativamente 
mayores ( $\mathrm{p} \leq 0.05)$ reportadas para el IV diluido 1:200 y $7.33 \mathrm{mg} / \mathrm{larva}$ ). estadio $(0.220 \mathrm{mg} / \mathrm{ml}$ para el extracto

Tabla 3

Concentración proteica soluble de los extractos de T. solanivora en cada estadio larvario con pesos iniciales promedio equivalentes a $120 \mathrm{mg}$ por muestra estudiada

Tecia solanivora

\begin{tabular}{cccc} 
Estadio & No. de & $\begin{array}{c}\text { Concentración proteica de los extractos } \\
(\mathbf{m g} / \mathbf{m l})\end{array}$ & $\begin{array}{c}\text { mg de } \\
\text { proteína/larva* }\end{array}$ \\
\cline { 3 - 3 } & larvas/extracto & Dilución 1:200 & 0.17 \\
\cline { 3 - 3 } I & 42 & 0.072 & 0.29 \\
II & 28 & 0.081 & 4.50 \\
III & 5 & 0.225 & 7.33 \\
IV & 3 & 0.220 &
\end{tabular}

\footnotetext{
* Corresponde al valor en mg de proteína soluble extraída por larva. El cálculo resulta de multiplicar la concentración proteica $(\mathrm{mg} / \mathrm{ml})$ de cada extracto por el factor de dilución 200, dividido para el número de larvas y multiplicado por $0.5 \mathrm{ml}$ que es el volumen del extracto.
}

Resultados similares se registraron en I, en contraste con las concentraciones S. tangolias, donde el menor valor proteico significativamente mayores $(\mathrm{p} \leq \quad 0.05)$ soluble se encontró en el extracto diluido reportadas para el extracto diluido 1:200 1:200 (0.096 mg/ml) del estadio III y en el (0.199 mg/ml) y para cada larva (3.98 $\mathrm{mg} /$ de cada larva (0.23 mg/larva) del estadio larva) del estadio IV.

Tabla 4

Concentración proteica soluble de los extractos de $S$. tangolias en cada estadio larvario con pesos iniciales promedio equivalentes a $120 \mathrm{mg}$ por muestra estudiada

\section{Symmetrischema tangolias}

\begin{tabular}{cccc} 
Estadio & $\begin{array}{c}\text { No. De } \\
\text { larvas/extracto }\end{array}$ & $\begin{array}{c}\text { Concentración proteica de los extractos } \\
(\mathbf{m g} / \mathbf{m l})\end{array}$ & $\begin{array}{c}\text { mg de } \\
\text { proteína/larva* }\end{array}$ \\
\cline { 3 - 3 } & 65 & Dilución 1:200 & 0.23 \\
I & 42 & 0.152 & 0.32 \\
II & 7 & 0.136 & 1.37 \\
III & 5 & 0.096 & 3.98 \\
IV & & 0.199 & \\
\hline
\end{tabular}

\footnotetext{
* Corresponde al valor en mg de proteína soluble extraída por larva. El cálculo resulta de multiplicar la concentración proteica $(\mathrm{mg} / \mathrm{ml})$ de cada extracto por el factor de dilución 200, dividido para el número de larvas y multiplicado por $0.5 \mathrm{ml}$ que es el volumen del extracto.
} 
Los análisis estadísticos ANOVA altamente significativos ( $p=0.000)$ (Figura detectaron diferencias altamente signifi1). Se encontraron diferencias $(\mathrm{p}=0.000)$ cativas, tanto a nivel de proteína soluble entre todos los valores promedio de halos total del extracto $(p=0.002)$, como a nivel medidos a las $8 \mathrm{~h}(8.2 \mathrm{~mm}), 24 \mathrm{~h}(9.43$ de concentración de proteína por larva de cada estadio y especie $(\mathrm{p}=0.000)$. $\mathrm{mm}), 48 \mathrm{~h}(12.68 \mathrm{~mm})$ y $72 \mathrm{~h}(16.58 \mathrm{~mm})$ de incubación. El tamaño promedio de los halos reportados para $S$. tangolias fue

Caracterización de la actividad más pequeño que el de T. solanivora, pero amilásica.- El análisis (ANOVA) de igualmente se encontraron diferencias la actividad amilásica de los extractos significativas $(\mathrm{p}=0.000)$ entre los intervalos proteicos obtenidos del estadio IV de de incubación, esto es, a las $8 \mathrm{~h}(3.33 \mathrm{~mm})$, T. solanivora y $S$. tangolias incubados a $24 \mathrm{~h}(4.29 \mathrm{~mm}), 48 \mathrm{~h}(9.42 \mathrm{~mm})$ y a las $72 \mathrm{~h}$ diferentes intervalos de tiempo fueron $(11.59 \mathrm{~mm})$, según se detalla en la Figura 1.

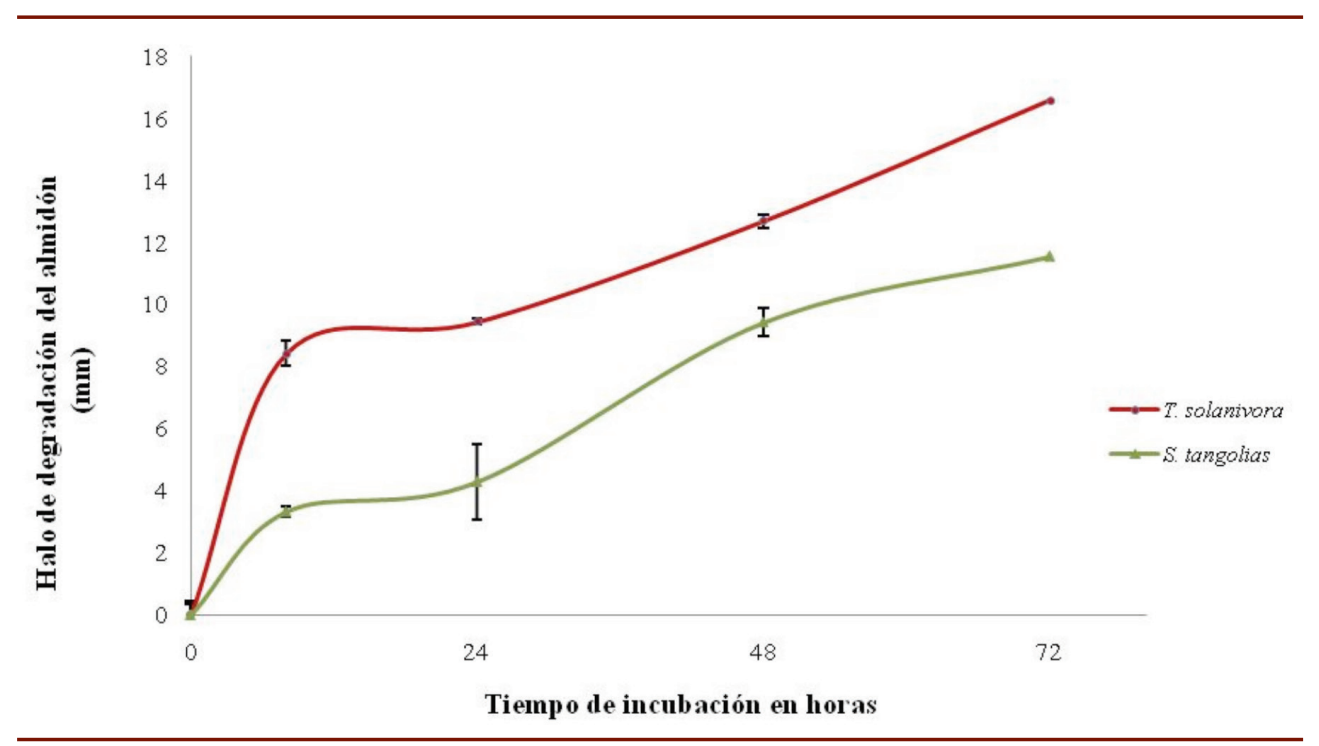

Figura 1. Actividad amilásica de los extractos de larvas del IV estadio de T. solanivora y de $S$. tangolias incubados en medio agar papa durante diferentes intervalos de tiempo ( 0 a $72 \mathrm{~h}$ ).

De la misma forma, se obtuvieron en cada una de las especies (Figura 2). diferencias altamente significativas T. solanivora presentó el menor halo de $(\mathrm{p}=0.000)$ para el análisis de los halos de degradación registrados a las $72 \mathrm{~h}$ de degradación del almidón en el III estadio incubación entre los estadios del I al IV, ocurrió con el extracto del IV estadio (16.58 
$\mathrm{mm})$. En los extractos de S. tangolias, el halo de menor degradación fue observado en la reacción del II estadio $(8.89 \mathrm{~mm})$ mientras que los halos más grandes ocurrieron con los correspondientes extractos de los estadios I y IV (13.42 y $11.54 \mathrm{~mm})$.
No existe una correlación entre la concentración proteica y la actividad amilásica entre las dos especies, los resultados arrojaron un $\mathrm{R}=0.176(\mathrm{p}=0.677)$ (Figura 2).

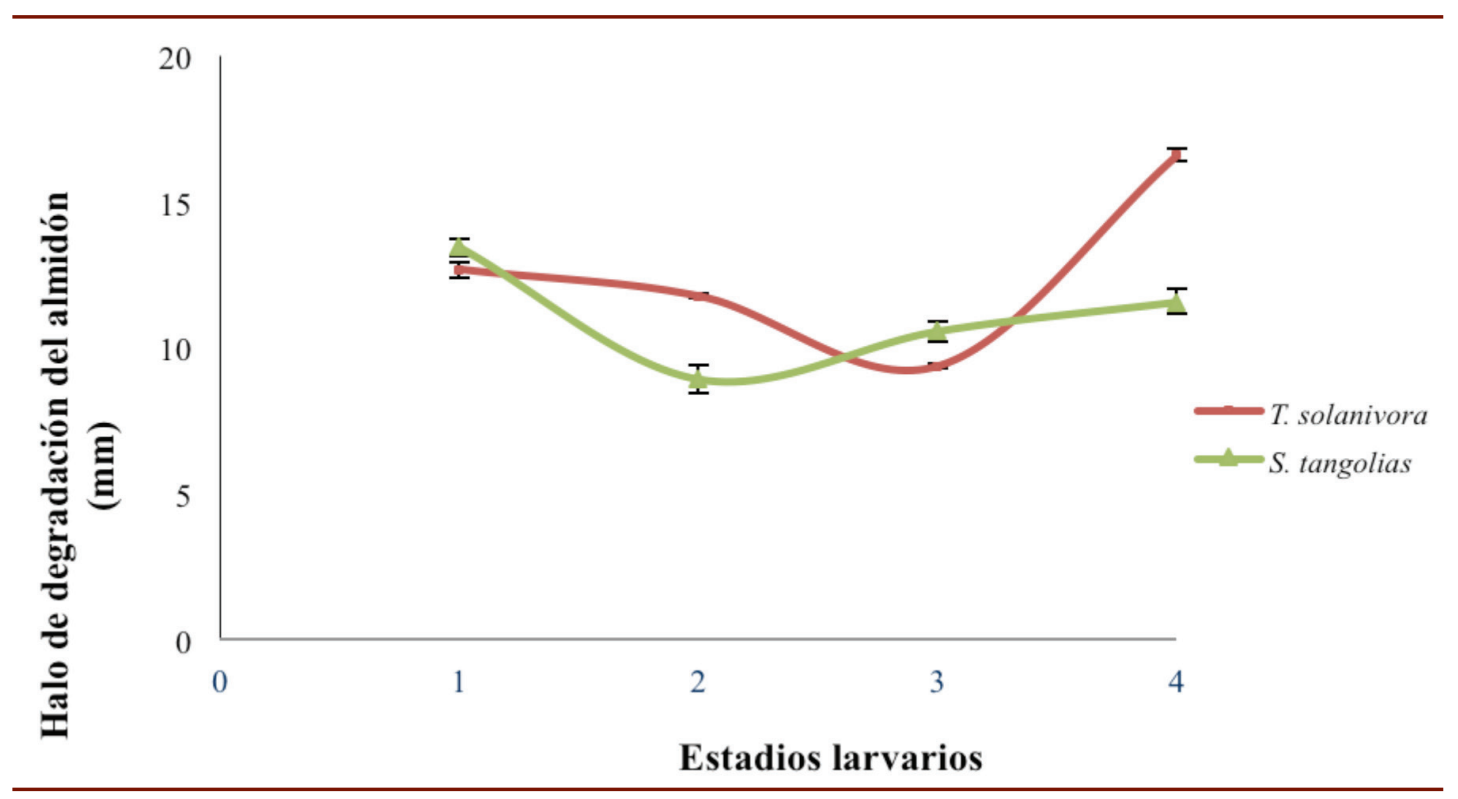

Figura 2. Actividad amilásica (halo de inhibición en $\mathrm{mm}$ ) de los extractos de larvas de los estadios I, II, III y IV de T. solanivora y de S. tangolias incubados en medio Agar Papa durante 72 h.

Separación e identificación de las presentes en los extractos correspondan proteínas solubles por electroforesis.- Se a miosina y anhidrasa carbónica, pudo distinguir la presencia de al menos 11 respectivamente. La proteína de nuestro bandas proteicas aparentemente similares interés, amilasa, fue identificada en ambas en los extractos de ambas polillas. Se especies (Figuras 3 y 4) según el peso estimaron pesos moleculares para las molecular referencial equivalente a $50 \mathrm{kDa}$ distintas bandas proteicas presentes en los sugerido y reportado para otros insectos de extractos, empleando proteínas estándares distintos géneros y especies (Ngernyuang con pesos moleculares entre 225 y 10 kDa. et al., 2010; Valencia-Jiménez et al., 2008; Basándonos en los pesos moleculares Baker, 1983). Se observó que tanto en T. calculados, creemos que es probable de solanivora (Figura 3) como en S. tangolias las bandas proteicas de $250 \mathrm{kDa}$ y $30 \mathrm{kDa}$ (Figura 4), la banda o bandas de alrededor 
de $50 \mathrm{kDa}$ detectadas en los extractos de los que las reportadas en los estadios III y IV, estadios I y II, fueron mucho más definidas donde aparecieron bastante difusas.

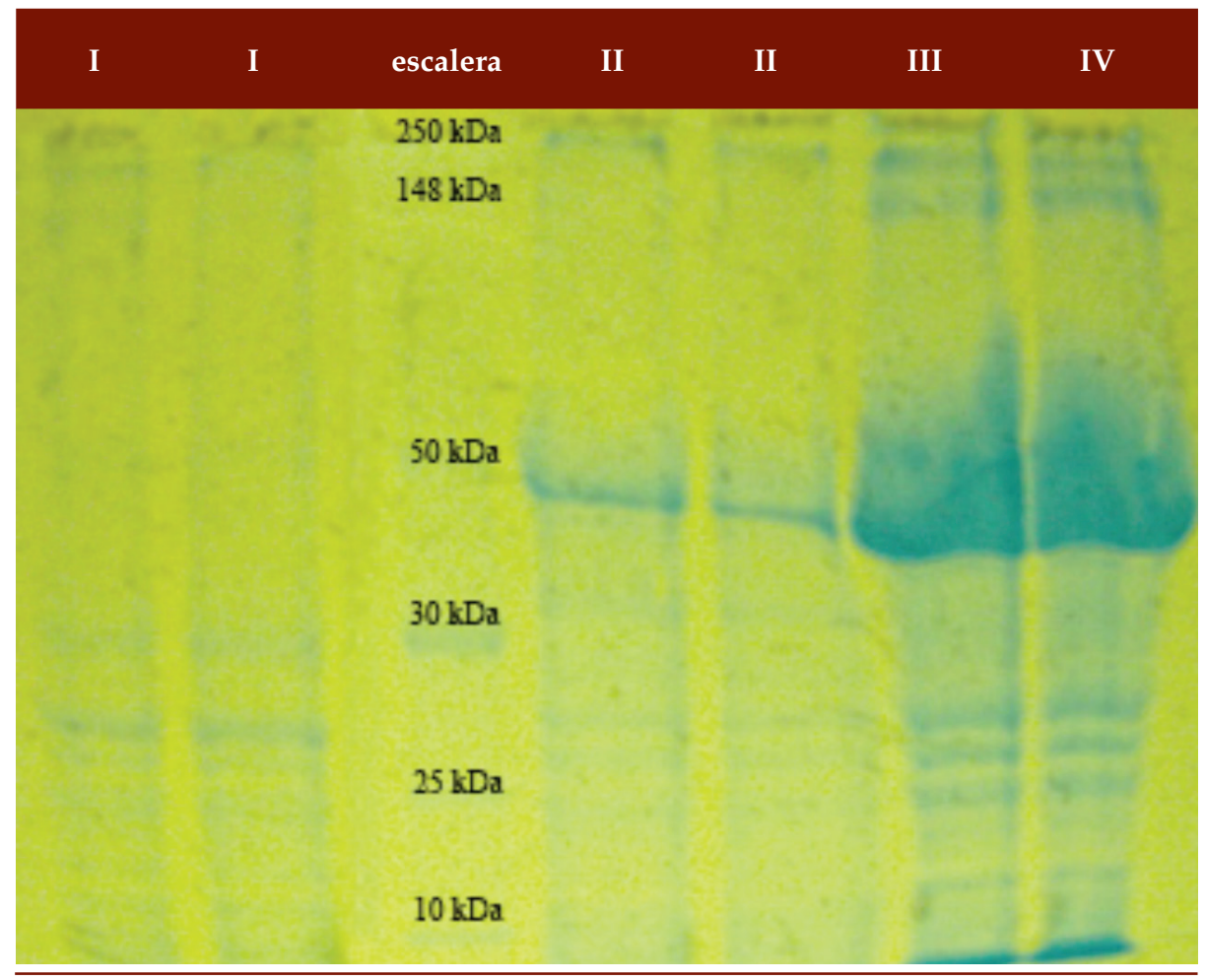

Figura 3. Electroforesis (SDS PAGE, gel $12 \%$ ) de los extractos larvarios de los estadios I, II, III y IV, de T. solanivora. Se incluye la escalera molecular.

\begin{tabular}{|c|c|c|c|c|c|c|c|c|}
\hline I & I & II & II & escalera & III & III & IV & IV \\
\hline & & & & $250 \mathrm{kDa}$ & & & & \\
\hline & & & & $148 \mathrm{kDa}$ & & & & \\
\hline & & & & $50 \mathrm{kDa}$ & & & & \\
\hline & & & & $30 \mathrm{kDa}$ & & & & \\
\hline & & & & $25 \mathrm{kDa}$ & & & & \\
\hline & & & & $10 \mathrm{kDa}$ & & & & \\
\hline
\end{tabular}

Figura 4. Electroforesis (SDS PAGE, gel $12 \%$ ) de los extractos larvarios de los estadios I, II, III y IV, de S. tangolias. Se incluye la escalera molecular. 


\section{DISCUSIÓN}

Con el fin de analizar comparativamente los extractos proteicos de los diferentes estadios larvarios de $T$. solanivora y $S$. tangolias, se contabilizaron un número de larvas requeridas para obtener un peso de $120 \mathrm{mg}$ para cada estadio. Los datos obtenidos demostraron que los cuatro estadios larvarios de $T$. solanivora representaban entre 1.5 y 1.9 veces más el peso correspondiente a los estadios de S. tangolias. Igualmente, se encontró que, en ambas especies, el peso del estadio I o III, al llegar al estadio II o IV, aumentó 1.5 veces su peso mientras que las larvas del estadio III pesaron alrededor de seis veces más que las larvas del estadio II (Tabla 2). Estos aumentos progresivos pero irregulares de la masa corporal son indicativos de cambios metabólicos estructurales y funcionales que acompañan a la singular madurez y a la capacidad infectiva de las polillas (Cadena et al., 2005; Herrera, 1998), cambios que parecen ser diferentes y únicos incluso para cada estadio lo que podría explicar por qué $T$. solanivora resulta ser muy agresiva (Vargas et al., 2004) y causa mayores daños que $S$. tangolias durante el proceso de infestación (resultados no reportados).

Al relacionar el peso larvario (Tabla 2) con el contenido de proteína soluble extraíble de cada estadio y en cada especie estudiada (Tabla 3 y 4), se observó que en $T$. solanivora la cantidad de proteína soluble extraída del estadio I solamente correspondió al $6 \%$ del peso de la larva, o al $7 \%, 17 \%$ y $16 \%$ del peso de los estadios II, III y IV. De igual forma, el porcentaje de proteína extraíble comparada con el peso total de cada larva de cada uno de los cuatro estadios de $S$. tangolias, fue de $13 \%, 11 \%, 8 \%$ y $17 \%$, respectivamente. Lo cual significaría que de los estadios I y II de S. tangolias, se extrajo proporcionalmente más proteína que aquella que se encontró en $T$. solanivora en los mismos estadios. No así en el IV estadio de $T$. solanivora donde la proteína soluble extraída fue casi el doble que la reportada para S. tangolias en el mismo estadio. Quizás estos resultados podrían ayudar a buscar diferencias en la manera de cómo la larva de cada especie ingresa al tubérculo y a explicar el mayor daño que ocasiona T. solanivora en contraste con S. tangolias).

Se reporta un aumento considerable de casi 16 veces el peso y 43 veces la cantidad de proteína soluble extraíble cuando $T$. solanivora maduró del estadio I al IV. S. tangolias, en cambio, con menores pesos y menor cantidad de proteína extraíble larvaria (según análisis entre estadios o por comparación entre las medias globales de la suma de los valores de los estadios), solamente mostró un aumento de casi 13 veces el peso y 17 veces la cantidad de proteína soluble extraíble cuando la larva pasó del estadio I al IV. Las diferencias significativas $(p \leq 0.05)$ en el peso y en la cantidad de proteína, son otros 2 parámetros que corroboran las diferencias estructurales y metabólicas entre especies así como la relativa similitud que podría existir para extraer con facilidad una fracción importante de varias proteínas solubles, algunas probablemente involucradas en la maduración de las larvas (Tabla 1) o en procesos de infestación únicos para cada 
una de estas plagas (Darvishzadeh et al., 2012; Kazzazi et al., 2005).

Reportes sobre la voracidad de estas polillas (Vargas et al., 2004) indican que una sola larva, al transformarse del estadio I al IV en aproximadamente 20 días (Tabla 1) consume entre 5 a $7 \mathrm{~g}$ de sustrato de papa. Para procesar esta cantidad de alimento, asumimos que la larva cuenta, entre otros, con enzimas como las amilasas, capaces de desdoblar los almidones en glucosas que se incorporarán al metabolismo celular.

El estudio de las amilasas se llevó a cabo en los extractos totales de cada estadio y de cada especie en consideración a los estudios de Kotkar et al. (2012) sobre la expresión genética de esta enzima en Lepidóptera la misma que varía para cada estadio, incluso en cada tejido comprometido: cabeza, cuerpo, vísceras, hemolinfa, donde no sería raro encontrar isoformas amilásicas específicas para un tejido determinado (Ngernyuang et al., 2010).

Las diferencias significativas en la actividad amilásica de los extractos ( $\mathrm{p} \leq 0.05)$, demostraron que estas enzimas continuaron su difusión y actividad en el sustrato de almidón aún después de varias horas a $18^{\circ} \mathrm{C}$ y a un pH óptimo de 7.8 (Figura 1). El pH en el cual se mantuvieron los extractos, al igual que la temperatura a la cual se realizaron los experimentos, parecen ser importantes parámetros en la activación y/o inhibición de las amilasas (Darvishzadeh et al., 2012; Valencia et al., 2008) porque afectan la conformación estructural de las mismas
(Álvarez, 2003). Observaciones previas (no reportadas) sobre el comportamiento de los extractos en distintos $\mathrm{pHs}$, justificaron el uso del tampón fosfato ( $\mathrm{pH}$ 7.8) el cual mantuvo la actividad y la estabilidad amilásica de los extractos a 18 y a $45^{\circ} \mathrm{C}$.

Además, la correlación $\quad(\mathrm{R}=0.176)$ obtenida cuando comparamos resultados ( $p>0.05)$ ) sugiere que la concentración proteica de los extractos solubles, no guardó relación ni geométrica ni logarítmica con la actividad amilásica en agar papa. En T. solanivora, mientras la proteína soluble aumentó logarítmicamente 43 veces del estadio I al estadio IV (Tabla 3), los halos promedio de degradación del almidón cambiaron erráticamente (Figura 2) en cada uno de los 4 estadios. Algo similar sucede con S. tangolias, mientras los extractos proteicos aumentaron 17 veces (Tabla 4), los halos de degradación (Figura 2) casi no cambiaron entre los estadios.

Estos resultados sugieren que la menor o mayor actividad amilásica medida como halos de degradación del almidón, no está proporcionalmente ligada a las diferentes concentraciones proteicas de los extractos, sino que, de acuerdo con estos experimentos, más bien se relacionan con las condiciones del sustrato, $\mathrm{pH}$, temperatura de reacción y a la singular expresión génica de cada estadio de desarrollo larvario (Ngernyuang et al., 2010; Valencia et al., 2008; Kazzazi et al., 2005).

La electroforesis en geles de poliacrilamida (SDS PAGE), hizo posible 
la separación de al menos 11 bandas proteicas bastante similares en cada estadio de ambas especies. Si bien no es posible identificar proteínas basándose únicamente en su patrón de migración electroforética, creemos que es razonable suponer que la banda de $250 \mathrm{kDa}$, presente en los extractos, corresponde a miosina asociada a la musculatura de cada estadio larvario. Asimismo, creemos que otra banda, con un peso molecular de aproximadamente 30 $\mathrm{kDa}$, podría corresponder a la anhidrasa carbónica. La banda correspondiente a esta proteína en los geles de poliacrilamida migró una distancia ligeramente menor en el gel con extractos de $T$. solanivora o ligeramente una mayor distancia en con extractos de $S$. tangolias, que el estándar de $30 \mathrm{kDa}$. Estas diferencias pudieran deberse a la existencia de isoenzimas, lo cual ha sido reportado previamente en otros insectos (Agudelo et al., 2010; Schwartz et al., 1993; Raymond, 1984; Childress y Sacktor, 1970).

Creemos que la amilasa corresponde a la banda de $50 \mathrm{kDa}$ en los geles de electroforesis, la cual está presente en los extractos de cada estadio en ambas polillas, (Figura 3 y 4). Dicha masa molecular es aproximadamente la que se ha reportado para otros insectos (Darvishzadeh et al., 2012; Ngernyuang et al., 2010; ValenciaJiménez et al., 2008 Kazzazi et al., 2005; Baker, 1991 y 1983).

El polimorfismo enzimático de las amilasas, en general, exhibe una interesante variabilidad funcional y se relaciona con el ambiente en el cual vive y evoluciona el insecto (Da Lage et al., 2002). En este contexto, los resultados sugieren que 1) la degradación de almidón aumenta en función del tiempo de contacto con la enzima, pero no es independiente de las concentraciones proteicas totales de los extractos, 2) que a las 72 h de reacción es más o menos similar en todos los estadios de las 2 especies aunque hay un ligero aumento en la actividad en el estadio IV de T. solanivora, 3) que la banda (s) detectada(s) en el rango de los $50 \mathrm{kDa}$ en los extractos de los estadios I y II, fue(ron) mucho más definida que las bandas difusas del III y IV estadios.

Se demuestra la presencia de amilasas en los cuatro estadios larvarios de las dos plagas estudiadas. Son necesarios estudios complementarios para la caracterización de estas enzimas, que permitan conocer las diferencias en la cantidad de proteínas extraibles, la regulación génica controlada de las enzimas (Janecek et al., 1997), la estructura proteica o la presencia de isoenzimas y el papel que estas desempeñan en los procesos de asimilación y digestión de almidones. Dicha información podría abrir las puertas para el desarrollo de nuevas medidas específicas e inéditas de biocontrol para plagas de este tipo.

\section{AGRADECIMIENTOS}

A la Pontificia Universidad Católica del Ecuador, por la asignación de fondos de investigación PUCE 2012-2013 y al Ingeniero Julio Sánchez-Otero, por el apoyo estadístico durante la realización de esta investigación. 


\section{REFERENCIAS BIBLIOGRÁFICAS}

Agudelo C D, Corena-Mcleo M y Robledo S M. 2010. Anhidrasa Carbónica de Plasmodium falciparum: un blanco útil para el diseño de medicamentos antimaláricos y compuestos bloqueadores de la transmisión de Malaria. Vitae, Revista de la Facultad de Química Farmacéutica, 17(1): 91-100.

Álvarez C. 2003. Actividad enzimática digestivayevaluación dedietas parael destete de larvas de la cabrilla arenera Paralabrax maculatofasciatus (Percoide: Serranidae). Tesis para obtener el grado de doctor en Ciencias Marinas. Instituto Politécnico Nacional, Departamento de Desarrollo de Tecnologías. Baja California, EEUU.

Astudillo Y y Soria C A. 2011. Identificación y caracterización de carbohidratos y proteínas en las secreciones del dorso y del pie del molusco Limax flavus. Revista Ecuatoriana de Medicina y Ciencias Biológicas, 22: 33-48.

Baker J E. 1991. Purification and partial characterization of $\alpha$ amylase allozymes from the lesser grain borer Rhyzorpertha dominica. Insect Biochemistry, 21: 303-311.

Baker J E. 1983. Properties of Amylases from midguts of larvae of Sitophilus zeamais and Sitophilus granarius. Insect Biochemistry, 13(4): 421-428.
Barragán A R. 2005. Identificación, Biología y comportamiento de las polillas de la papa en el Ecuador. PROMSAMAG, PUCE. 12 pp. ISBN 9978-77124-7. Quito, Ecuador.

Cadena M, Naranjo A, Nústez C E. 2005. Evaluación de la respuesta de 60 genotipos de Solanum phureja (Juz. et Buk.) al ataque de la Polilla guatemalteca (Tecia solanivora Povolny). Agronomía Colombiana, 23 (1).

Campos F A P, Xavier-Filho J, Sila C P y Ary M B. 1989. Resolution and partial characterization of proteinases and amylases from midguts of larvae of the Bruchid Beetle Callosobruchus maculates (F). Comparative Biochemistry and Physiology, 92B(1): 51-57.

Childress C C y Sacktor B. 1970. Regulation of glycogen metabolism in insect flight muscle. Purification and properties of phospholylases in vitro and in vivo. The Journal of Biological Chemistry, 245: 2927-2936.

Da Lage J L, Wormhoudt A V y Cariou M L. 2002. Diversity and in animals, Review, Biologia, Bratislava, 57(11): 181-189.

Darvishzadeh A, Bandani A R, Karimi J y Timouri G. 2012. Biochemical characterisation of digestive $\alpha$-amylase of Red Palm Weevil, Rhynchophorus ferrugineus (Olivier, 
1790) (Coleoptera: Curculionidae), Archives of Phytopathology and Plant Protection, 45(18): 2132-2142.

Domínguez J, Carrero C, Ramírez W, Segovia Py Pino, H. 2009. Evaluación del efecto de insecticidas sobre larvas de Tecia solanivora. Agricultura Andina, 17: 61-73.

Herrera M. 2010. Interacciones intra e interespecíficas entre polillas de la papa (Lepidoptera: Gelechiidae). Tesis de Licenciatura en Ciencias Biológicas, Pontificia Universidad Católica del Ecuador. Quito, Ecuador.

Herrera F. 1998. La polilla guatemalteca de la papa. Biología, comportamiento y prácticas de manejo integrado. CORPOICA. Programa Regional Agrícola. p. 14.

Janecek S, Svensson B y Henrissat B. 1997. Domain evolution in the a-amylase family. Journal of Molecular Evolution, New York, 45: 322-331.

Kazzazi M, Bandani A R y Hosseinkhani S. 2005. Biochemical characterization of $\alpha$-amylase of the Sunn pest Eurygaster integriceps. Entomological Science, 8, 371-377.

Kotkar H M, Bhide A J, Gupta V S y Giri A P. 2012. Amylase gene expression patterns in Helicoverpa armigera upon feeding on a range of host plants. Elsevier, Gene 501: 1-7.
Lagnaoui A, Cañedo V y Douches S D. 2000. Evaluation of Bt-cry1Ia1 (cryV) transgenic potatoes on two species of potato tuber moth, Phthorimaea operculella and Symmetrischema tangolias (Lepidoptera: Gelechiidae) in Peru. CIP Program Report 19992000, 117-121.

Ngernyuang N, Kobayashi I, Promboon A, Ratanapo S, Tamura T y Ngernsiri L. 2010. Cloning and expression analysis of the Bombyx mori $\square$-amylase gene (Amy) from the indigenous Thai silkworm strain, Nanglai. Journal of Insect Science, 11(38): 1-16.

Raymond P H. 1984. The role of carbonic anhydrase in blood ion and acidbase regulation. American Zoologist, 24(1): 241-251.

Rodríguez-Guerra A, Barnes C, Ordóñez M E, Salazar A, Soria CA. 2012. Identificación y evaluación de algunos hongos con actividad celulásica aislados en Ecuador. Revista Ecuatoriana de Medicina y Ciencias Biológicas, 23: 65-81.

Schwartz L, Jones M, Kosz L, Kuah K. 1993. Selective repression of actin and myosin heavy chain expression during the programmed death of insect skeletal muscle. Developmental Biology, 158(2): 448-455.

Trujillo E, Ríos D, Cabrera R. 2002. Distribución de Tecia solanivora 
(Polvoni) (Lepidoptera: Gelechiidae) en Tenerife, Islas Canarias, España. Avances en investigación y manejo integrado de la polilla gualtemalteca de la papa, Tecia solanivora. Memorias del II taller Internacional de polilla gualtemalteca, Quito, Ecuador, 191192.

Valderrama A M, Velásquez N, Rodríguez E, Zapata A, Zaidi M Z, Altosaar I y Arango R. 2007. Resistence to Tecia solanivora in three transgenic Andean varieties of potato expressing Bacillus thuringiensis Cry1Ac protein. Journal of Economic Entomology, 100(1): 172-179.

Valencia-Jiménez A, Arboleda V, López Ávila M F y Grossi-de-Sá. 2008. Digestive alpha-amylases from Tecia solanivora larvae (Lepidoptera:
Gelechiidae): response to $\mathrm{pH}$, temperature and plant amylase inhibitors. Bulletin of Entomological Research, 98: 575-579.

Vargas B I, Rubio S A y López-Avila A. 2004. Estudios de hábitos y comportamiento de la polilla guatemalteca Tecia solanivora (Lepidoptera: Gelechiidae) en papa almacenada. Revista Colombiana de Entomología, 30(2): 1-4.

Viktorinova I, Kucerova L, Bohmova M, Henry I, Jindra M, Dolezal P, Zurovcova M y Zurovec M. 2011. Characterization of two closely related a-amylase paralogs in the bark beetle, Ips typographus (L.). Archives of Insect Biochemistry and Physiology, 77(4): 179-198. 